\title{
El Impacto de la Acreditación en la Mejora de la Calidad de los Programas Educativos que Ofrece la Universidad Autónoma de Tamaulipas: Un Estudio de Caso
}

\section{The Impact of Accreditation on Improving the Quality of Educational Programs Offered by the Autonomous University of Tamaulipas: A Case Study}

\author{
Erik Márquez de León *1 \\ Zulma Raquel Zeballos Pinto ${ }^{2}$ \\ ${ }^{1}$ Universidad Autónoma de Tamaulipas \\ ${ }^{2}$ Universidad de Guadalajara
}

\begin{abstract}
El objetivo de esta investigación consiste en buscar evidencias sobre si la acreditación ha tenido un impacto significativo en la mejora de la calidad de los programas educativos que ofrece la Universidad Autónoma de Tamaulipas, y si este proceso ha impulsado cambios institucionales para hacer sustentable la calidad educativa, ampliar la responsabilidad social de la institución, producir conocimiento para el desarrollo sustentable y contribuir al tránsito hacia una de las sociedades del conocimiento en Tamaulipas. Se trata de un estudio de caso con enfoque cuantitativo y alcance descriptivo. De acuerdo al muestreo, se analizó 10 (24\%) programas educativos acreditados en distintos campos del conocimiento. Para ello fue necesario, conocer la percepción de los directivos/gestores, profesores de tiempo completo y estudiantes de los programas educativos acreditados seleccionados como representativos de la universidad, a través de un cuestionario diseñado para cada uno de estos actores en relación al impacto de la acreditación en la mejora de la calidad de los programas académicos de grado en los que se desenvuelven. Considerando algunos aspectos tales como: calidad, calidad educativa, gestión institucional y gestión de la calidad. Los resultados y conclusiones muestran que la percepción de los actores indica que la acreditación ha tenido un impacto parcial en los programas educativos.
\end{abstract}

Palabras clave: Educación superior, Programas educativos, Acreditación, Impacto, Mejora de la calidad, Calidad educativa.

The objective of this research is to look for evidence on whether accreditation has had a significant impact on improving the quality of educational programs offered by the Autonomous University of Tamaulipas, and if this process has prompted institutional changes to make educational quality sustainable, expand the social responsibility of the institution, producing knowledge for sustainable development and contribute to the transition to a knowledge society in Tamaulipas. It is a case study with a quantitative approach and inferential descriptive scope. In the sample, 10 (24\%) accredited educational programs in different fields of knowledge were analyzed. In order to do this, it was necessary to know the perception of executives / managers, full-time teachers and students of accredited educational programs selected as representative of the university through a questionnaire designed for each of these actors regarding the impact of accreditation in the improvement of the quality of their respective academic degree programs. Aspects such as quality, educational quality, institutional management and quality management were considered. The findings and conclusions show that the perception of the actors indicates that accreditation has had a partial impact on educational programs.

Keywords: Higher education, Educational programs, Accreditation, Impact, Improvement of the quality, Quality of education.

*Contacto: ermarquez@uat.edu.mx

issn: 1989-0397

www.rinace.net/riee/

https://revistas.uam.es/riee
Recibido: $\quad 8$ de julio de 2016

$1{ }^{\text {a }}$ Evaluación: 12 de noviembre de 2016

$2^{\text {a }}$ Evaluación: 14 de diciembre de 2016

Aceptado: 29 de diciembre de 2016 


\section{Planteamiento y justificación del problema}

Las políticas de organismos internacionales como la Organización para la Cooperación y Desarrollo Económicos, el Banco Mundial, la Organización de las Naciones Unidas para la Educación, la Ciencia y la Cultura, establecen la necesidad de convergencia y ajuste entre los países en un período en el que está en juego la propia esencia de la relación entre la institución universitaria y la sociedad.

Los cambios en el entorno, el conocimiento y las necesidades sociales hacen que el sector universitario necesite una profunda transformación que permita atender las demandas de la sociedad, como las del sector productivo, satisfacer las expectativas que tienen los estudiantes y sus familias sobre lo que la universidad puede aportarles y responder a las exigencias de los agentes financiadores, particularmente en el uso eficiente de los recursos económicos y la aplicación de sistemas de control y rendición de cuentas.

El discurso de la transformación de la universidad debe darse a través de la calidad educativa. De acuerdo al Plan de Acción para la transformación de la Educación Superior en América Latina y el Caribe, suscrito en el marco de la Conferencia Mundial sobre Educación Superior, considera a la calidad como "la adecuación del ser y quehacer de la educación superior a su deber ser" (UNESCO, 1998, p. 4).

El tema de la calidad en la educación superior ocupa un lugar destacado en la discusión de las políticas públicas para la educación mexicana para las tres últimas décadas, teniendo como elemento fundamental la evaluación y la acreditación. La evaluación y la acreditación constituyen un curso de acción para el Sistema Nacional de Educación Superior. La evaluación entendida como "un sistema de coherencias entre los distintos factores que constituyen el ser, el hacer y el deber ser de las instituciones de educación superior" (ANUIES, 2000, p. 72).

La acreditación concebida como "un proceso de evaluación que culmine con un reconocimiento público a la labor que realiza una institución” (Marúm, 1995) o bien como "una rendición de buenas cuentas a la sociedad, como responsabilización institucional de los resultados obtenidos con los recursos asignados a la educación superior" (Marúm, Mustre y Nieto, 2011). La acreditación se ha utilizado como una estrategia de planeación de las Instituciones de Educación Superior (IES), y es considerado un medio que permite alcanzar la calidad educativa. Las IES buscan gestionar la calidad educativa por medio del enfoque del aseguramiento de la calidad, sin embargo, estudios demuestran que este enfoque no es el adecuado para el ámbito educativo. El enfoque de la mejora de la calidad de acuerdo a sus características es el más adecuado para la gestión universitaria (Cardona, 2011).

En el Segundo Congreso Internacional Acreditación y Certificación de Programas e Instituciones de Educación Superior (CIAC, 2012) se presentaron una serie de trabajos referentes al impacto de la acreditación en la mejora continua de la calidad de los programas educativos en distintas universidades del país. Los resultados de estas investigaciones muestran la necesidad de seguir demostrando con evidencias los beneficios de la acreditación en la mejora de la calidad de los programas académicos. La acreditación ha logrado cumplir parcialmente con las expectativas que se tenía sobre su impacto en la calidad de la educación superior, de ahí la importancia de seguir realizando estudios de caso en otras universidades del país con la finalidad de presentar evidencias 
que permitan mostrar los avances que ha generado la acreditación a raíz de su implementación en la educación superior.

En este contexto, se ha elegido la Universidad Autónoma de Tamaulipas (UAT), como un caso de estudio, en el cual se analiza los cambios o transformaciones que se han llevado a cabo en la mejora de la calidad de los Programas Educativos (PE) acreditados. Lo anterior se aborda teniendo como punto de referencia las siguientes preguntas de investigación ¿Cuál ha sido el impacto de la acreditación en la mejora de la calidad de los PE de la UAT? ¿El actual modelo de acreditación ha impulsado la mejora en la calidad educativa en los PE analizados? Se parte de tres hipótesis: de investigación (impacto significativo), nula (no existe impacto) y alternativa (existe un impacto parcial) de la acreditación para la mejora de la calidad de los programas acreditados.

\section{Fundamentación teórica}

Se han realizado diversos estudios de caso sobre los impactos de los sistemas de aseguramiento de la calidad en Latinoamérica y resto del mundo. Durante el período 2009-2012, se desarrolló el Proyecto Alfa III "Aseguramiento de calidad: políticas públicas y gestión universitaria” con la colaboración de investigadores de distintas IES de América Latina y Europa, participaron universidades de México, Argentina, Colombia, Costa Rica, Chile, España y Portugal. El trabajo analizó la eficiencia y pertinencia de las políticas públicas basadas en la evaluación y acreditación como procesos orientados a la mejora de la calidad. De acuerdo al Centro Interuniversitario de Desarrollo (CINDA, 2012) el proyecto fue de carácter exploratorio y de percepción de impactos, se llevó a cabo en dos momentos, el primero de orden teórico para dar sentido a la percepción de los impactos de los mecanismos de aseguramiento de la calidad y el segundo momento relacionado con los estudios de caso.

Para el caso mexicano, según Buendía (2014) se eligieron las universidades de acuerdo a la clasificación de regiones que utilizada la Asociación Nacional de Universidades e Instituciones de Educación Superior (ANUIES). Las instituciones participantes fueron: Universidad Autónoma de Sinaloa (Noroeste) con Derecho, Universidad de Guadalajara (Centro-Occidente) con Ingeniería Electrónica, Universidad Autónoma Metropolitana (Metropolitana) con Matemáticas, Universidad Autónoma Chapingo (Centro-Sur) con Agronomía y la Universidad Veracruzana (Sur-Sureste) con Medicina. Las categorías genéricas explicativas fueron la evaluación y acreditación, las variables transversales de que se analizaron son la estructura organizacional y la gestión institucional. Los efectos del cambio organizacional e institucional se identificaron a partir de la valorización del cambio en los planes y programas de estudio, procesos de aprendizaje, infraestructura y docencia, innovación, así como el sistema de reglas, normas e incentivos.

Los resultados dan evidencia de las percepciones sobre los efectos de la evaluación y acreditación de diferentes actores involucrados. En cuanto a la estructura organizativa y gestión institucional se logró observar que en todos los casos la construcción de una estructura paralela de gobierno de las universidades donde se definen las decisiones más relevantes, ante una aparente incapacidad institucional de las universidades para generar sus propias estrategias para mejorar su desempeño y la calidad de sus procesos académicos y de gestión. Las IES con una dinámica propia de cambios organizacionales 
tienen una mejor capacidad de respuesta a las políticas de evaluación y de acreditación. Además, los procesos de evaluación y acreditación no muestran efectos contundentes de mejora en la calidad de los PE, esta depende de la concurrencia de muchos factores y no sólo es determinada por esos procesos. Los procesos de cambio organizacional e institucional surgen con dinámicas y velocidades distintas. En algunos casos el cambio surge de las mismas organizaciones y en otros depende de la influencia de elementos externos que hacen presión sobre sus estructuras para moverse de su inercia. Los maestros en general consideran que los procesos de evaluación y acreditación no han tenido impacto en los planes y programas de estudio, los efectos en los procesos de aprendizaje son menos visibles, no implica una modificación sustancial de éstos procesos, los efectos se quedan en la parte formal y operativa organizacional. Tampoco se aprecian efectos contundentes en los procesos de innovación didáctica.

Existen otros estudios como "El impacto de la evaluación externa en dos instituciones de educación superior en México" (Castillo, 2004) que analiza el cambio institucional universitario desde la perspectiva desde la política mexicana de educación superior en cuanto al financiamiento y la calidad, para verificar la centralidad de dicha política como el principal detonador del cambio institucional en el ámbito de las universidades. Se concluye que el impacto de la evaluación externa se observa en la actualización de planes de estudio y en menor medida en la gestión de la calidad educativa.

Por su parte, De la Garza (2013) realizó el estudio "La evaluación de programas educativos del nivel superior en México. Avances y perspectivas" concluyendo que "la evaluación externa y la acreditación de la educación superior han logrado superar inercias, inconformidades y obstáculos diversos, y han perfeccionado sus estrategias, metodologías y procedimientos" (p. 42). Se percibe un notable avance en la cultura de la evaluación y sobre los inconvenientes entre la autonomía universitaria en relación con la evaluación y acreditación. Además de reconocer que las políticas públicas han proporcionado un impulso considerable a ambos procesos.

De los estudios revisados, el proyecto del CINDA en México fue uno de los más importantes en materia de impactos y percepción sobre el aseguramiento de la calidad en programas educativos, de ahí la relevancia que tienen los resultados para comparar lo sucedido en otros escenarios, como es el caso de la Universidad Autónoma de Tamaulipas. Una vez consultado el estado de la cuestión en relación al objetivo de la investigación, fue necesario analizar los referentes teóricos conceptuales y enfoques relacionados con calidad educativa vía los procesos de acreditación de programas universitarios para conocer y comprender desde dónde y cómo se ha estudiado el impacto de la acreditación en la mejora de la calidad de la educación superior.

La gestión de la calidad tiene dos enfoques, uno es el aseguramiento de la calidad, también conocido como gestión de la calidad total (TQM) y otro es la mejora de la calidad. Ambos enfoques difieren mucho en sus características y maneras de llevarse a cabo en las instituciones. $\mathrm{El}$ aseguramiento de la calidad pretende que los problemas de la educación se resuelvan de un modo parecido a como se solucionan en la producción industrial, por la vía del establecimiento de normas que incidan en los procesos educativos conforme a criterios ya establecidos. Es por ello que en este enfoque la calidad se vincula a la eficiencia en el uso de recursos que se ponen al servicio de una finalidad y, por tanto, la labor de la gestión consiste en establecer procedimientos que al 
aplicarse garantizan la relación entre recursos y fines, y en asegurar su ejecución. Esta forma de concebir a la calidad se acerca a dos de las concepciones de calidad establecidas por Harvey y Green (1993) como excepcional con noción de excelencia y como adecuación a una finalidad.

El segundo enfoque confiere al docente la libertad de definir sus objetivos de calidad y diseñar el proceso de aprendizaje, prescindiendo de las normas de procedimientos, incluso, y en última instancia, de las que se interpretan como parte de la profesión del docente. Este planteamiento confía en la capacidad del docente de poner en marcha un proceso acumulativo, en el que el agente de cambio, es decir, el propio docente, individual o colectivamente, formula un proceso de actuación que válida progresivamente con los resultados de su trabajo, en un proceso de mejora continua sobre el que los evaluadores ejercen un papel meramente de asesor.

Estos enfoques, se conducen en direcciones opuestas y, lo que es más importante, es visible que se encuentren con respuestas completamente diferentes por parte de quienes se encuentran en las instituciones universitarias, y en particular por parte de los docentes. Cardona (2011) los denomina enfoque retrospectivo (aseguramiento de la calidad) porque su análisis se basa en lo que ocurrió en el pasado, y enfoque prospectivo (mejora de la calidad), porque su análisis se fundamenta en el presente y se orienta en el futuro.

Contemplando a la acreditación de PE desde los dos enfoques de la gestión de la calidad, se observa que existe una gran diferencia en la forma en que se lleva a cabo este proceso de evaluación y en la forma en que los miembros de las IES responden al proceso y a la retroalimentación de sus resultados.

Contrastando el enfoque del aseguramiento de la calidad en los procesos de acreditación, se encontró aspectos muy particulares como el cumplimiento de estándares externos que se evalúan bajo un concepto de auditoría donde el principal instrumento es la evaluación externa que asegure la eficiencia en el uso de los recursos y la eficacia en los resultados. El evaluador externo llega con una serie de indicadores que debe de cumplir el programa educativo para poder ser nombrado acreditado y por ende de calidad. En este sentido, el proceso de acreditación se vuelve un check list, predominando el análisis cuantitativo.

En los procesos evaluación se corre el riesgo que las instituciones educativas se centren solamente en cumplir con los indicadores (Brennan, 1998), esto pudiera llevar a malas prácticas que ponen en riesgo el objetivo real de la evaluación que son cambiar y mejorar. Habrá que agregar que los resultados que emite el proceso de acreditación adoptan un estilo inquisitivo en las observaciones y emiten alternativas de solución a los problemas encontrados, de forma estricta y directa. Entonces los responsables de guiar los procesos en las IES establecen acciones puntuales y con plazos bien determinados para solventarlas y la evaluación la llevan a cabo de arriba hacia abajo, sin oportunidad de retroalimentación ni búsqueda de opiniones o comentarios de abajo hacia arriba. Al puro estilo de la gestión industrial taylorista.

El sentido de la calidad que impera en el ámbito universitario, sigue siendo vista por el número de clases, de estudiantes graduados, del nivel de educación de los estudiantes, del número de conferencias impartidas, de textos publicados, de participación en grupos de trabajo académico, entre otros. En efecto cuestiones de contabilidad y compensación 
económica, esta forma de pensar sigue presente en los ámbitos universitarios (Mantilla, 2006).

Según Marúm (2012) el enfoque que ha prevalecido en los procesos de acreditación y certificación es el enfoque del aseguramiento de la calidad en América Latina y de forma particular en México es:

Lo que ha reforzado los modelos de gestión industrial que obstaculizan el paso hacia la gestión del conocimiento y las respuestas que espera y merece la sociedad para transitar hacia una de las sociedades del conocimiento y para asegurar la sustentabilidad de su desarrollo, pues este modelo de calidad limita su capacidad de resolver problemas complejos y su capacidad y facultad de adaptar su estructura y funcionamiento a las necesidades y situaciones cambiantes que se le van planteando en un entorno complejo y de vertiginosas transformaciones. (Marúm, 2012, p. 20)

Si las universidades logran ver a la acreditación desde el enfoque de la mejora de la calidad, por sus características ya antes mencionadas seguramente la acreditación contribuiría a impulsar cambios institucionales para hacer sustentable la calidad educativa, ampliar el compromiso social responsable de la institución, producir conocimiento para el desarrollo sustentable y contribuir al tránsito hacia una de las sociedades del conocimiento.

En este contexto la acreditación "se ha dedicado a fomentar procesos y normas que se han establecido para gestionar la calidad estandarizada” (Marúm, 2011, p. 42) y otra de sus funciones ha sido certificar la calidad en el desarrollo de ciertas funciones de la institución. Las normas establecidas en la organización emergen en gran medida a la influencia del proceso de acreditación ya que desde un primer momento se estandarizan los indicadores por dimensiones que deben cumplir las IES y que indudablemente tanto la gestión institucional como la gestión de la calidad buscan responder a las estandarizaciones de la acreditación.

La evaluación de la calidad y su reconocimiento público con instrumentos como la acreditación han penetrado la cultura académica, sin embargo, resulta que la evaluación de la calidad progresivamente se transforma mucho más en una herramienta de control que una herramienta de mejora, siendo este el valor prioritario de los profesores (Días, 2012). Para que la acreditación opere como un proceso de mejora de la calidad requiere de utilizar instrumentos no estandarizados que vean más allá de indicadores únicos y visualicen la calidad en contextos diversificados con características diferentes.

Como bien menciona Lemaitre (2012) "el objetivo de mejoramiento enfatiza el hecho de que la responsabilidad por la calidad corresponde a las instituciones; por tanto, se centra en su capacidad para desarrollar y aplicar políticas y mecanismos eficaces de autorregulación y mejora continua" (p. 5). Los indicadores se responden priorizando la reflexión e incorporación de experiencias, investigaciones y resultados de lo que se está haciendo para el logro de la misión, teniendo en cuenta las condiciones de viabilidad del modelo de calidad seguido, esto es la base de la mejora. Se trata de valorar la parte cualitativa. Los evaluadores externos asumen un papel de colaboradores y no de auditores, dispuestos a orientar y ayudar a la institución emitiendo observaciones que dejan a consideración de los miembros de la organización quienes se basaran en la reflexión y análisis institucional. Los gestores de las IES buscan atender a las observaciones realizadas en el proceso de acreditación de manera abierta, es decir tomando en cuenta a todos los miembros de la institución, y forma constructiva 
orientándose hacia el futuro. El proceso se vuelve participativo pues se realiza de abajo hacia arriba propiciando el trabajo colaborativo.

En contraparte debemos ver a la calidad "como una construcción social hecha por actores, instituciones y políticas que funcionan bajo contextos económicos, sociales y políticos específicos, en los ámbitos local, regional, nacional e internacional" (Cardona, 2011, p. 21). La calidad debe tomar en cuenta la naturaleza de las instituciones, así como el contexto social. La calidad la construyen los miembros de las IES de acuerdo a sus necesidades tanto al interior como al exterior, se trata de lograr los fines propuestos en la naturaleza y misión de la institución educativa cuya finalidad última es la transformación positiva de sus estudiantes. La calidad se construye tomando en cuenta las opiniones de todos los miembros de la universidad de abajo hacia arriba.

El enfoque que debe prevalecer en los procesos de acreditación es el de la mejora de la calidad partiendo de la construcción social de la misma (Cardona, 2011) atendiendo a las necesidades del contexto logrando los fines propuestos en la naturaleza y misión de la institución educativa cuya finalidad última es la transformación positiva de sus estudiantes, generando la readaptación continua de la institución a su multientorno social, cultural y político (Latapí, 2008). La acreditación "debe estar ligada a la misión social de la universidad, mediante un profundo cuestionamiento permanente de la vida cotidiana de la organización, centrada en el estudiante y como el bien común" (Rosario, 2012, p. 206). La universidad tiene que apoyarse en la acreditación como una estrategia para mejorar la calidad de sus programas académicos y atender los aspectos esenciales de la calidad como los son el alumnado, el profesorado y el aprendizaje.

Siguiendo a Marúm, la gestión institucional debe ser asumido como un proceso que integra el trabajo humano, y de una manera lógica y racional incluye a los integrantes de una institución para realizar actividades que permitan cumplir con los fines y objetivos institucionales, en el que predomine la burocracia profesional, como parte de la estructura organizacional. Desde la gestión de la calidad se debe fomentar la acreditación como "procesos acordes con su naturaleza dinámica y autogestora para lo que se requiere estructuras y procesos diferentes a los procesos y normas que se han establecido para gestionar la calidad estandarizada” (Marúm, 2011, p. 42).

El impacto de la acreditación en la gestión de la calidad para la mejora de la calidad de los programas educativos debe mostrar que las acciones que desarrolla la universidad están centradas en formar de los estudiantes tanto en lo profesional como en la parte ciudadana, impulsar la formación docente y dar prioridad a la práctica docente, investigación y responsabilidad social. Entonces sí, la acreditación estará en condiciones de contribuir a impulsar cambios institucionales para hacer sustentable la calidad educativa, ampliar el compromiso social responsable de la institución, producir conocimiento para el desarrollo sustentable y contribuir al tránsito hacia una de las sociedades del conocimiento.

\section{Objetivos}

Buscar evidencias sobre si la acreditación ha tenido un impacto significativo en la mejora de la calidad de los Programas Educativos que ofrece la Universidad Autónoma de Tamaulipas, y si este proceso ha impulsado cambios institucionales para hacer 
sustentable la calidad educativa, ampliar el compromiso social responsable de la institución, producir conocimiento para el desarrollo sustentable y contribuir al tránsito hacia una de las sociedades del conocimiento en Tamaulipas.

\section{Metodología}

Para la construcción de la calidad y calidad educativa se tomó como referentes teóricos metodológicos a Harvey y Green (1993), Cruz, Escrigas, López, Sanyal y Tres (2007), Latapí (2008), Marúm (2011), Cardona (2011), mientras que para la gestión institucional los referentes fueron Mintzberg (1997) y Senge (2002), finalmente para la gestión de la calidad se consideró a Drucker (2004), Fernández (2004), Dias (2012), Marúm (2012) y Oloskoaga (2012). Con base en estos referentes, se realizó un estudio de caso con enfoque cuantitativo y alcance descriptivo, se aplicó la técnica de la encuesta y el instrumento del cuestionario. Las preguntas integraron respuestas de opción múltiples, denominado diferencial semántico, los encuestados pueden elegir, subrayando: mucho, poco o nada. Aunque los tres tipos de cuestionarios presentan esta misma estructura, las preguntas fueron planteadas de manera distinta.

Respecto a la muestra, en una primera etapa se aplicó el muestreo intencional, el cual da la facultad al investigador bajo estricto juicio personal seleccionar los elementos muestrales representativos del universo (Namakforoosh, 2002), bajo esta premisa se seleccionó 10 de $41 \mathrm{PE}$ acreditados de la zona universitaria con mayor relevancia en la UAT, la zona centro. En una segunda etapa, se aplicó el muestreo estratificado al conjunto de PE identificados como representativos, donde se consideró tres poblaciones (tabla 1): directores/gestores, profesores y estudiantes, tratándose de un muestreo que busca incrementar el grado de precisión muestral, "la población o universo se divide en segmentos y se selecciona una muestra para cada segmento" (Hernández, Fernández y Baptista, 2010, p. 180).

Tabla 1. Población

\begin{tabular}{|c|c|c|c|}
\hline Programa Educativo & $\begin{array}{l}\text { DIRECTORES } \\
\text { /GESTORES }\end{array}$ & $\begin{array}{l}\text { PROFESORES } \\
\text { (PTC) }\end{array}$ & ESTUDIANTES \\
\hline Licenciado en Derecho & 6 & 24 & 1,341 \\
\hline Licenciado en Enfermería & 6 & 20 & 1,341 \\
\hline Licenciado en Psicología & 6 & 6 & 986 \\
\hline Licenciado en Trabajo Social & 6 & 18 & 797 \\
\hline Contador Público & 6 & 19 & 873 \\
\hline Licenciado en Ciencias de la Comunicación & 6 & 25 & 521 \\
\hline $\begin{array}{l}\text { Licenciado en CC. de la Educación con } \\
\text { opción en Tecnología Educativa }\end{array}$ & 6 & 14 & 569 \\
\hline Licenciado en Administración & 6 & 13 & 568 \\
\hline Médico Veterinaria y Zootecnia & 6 & 48 & 539 \\
\hline $\begin{array}{l}\text { Licenciado en CC. de la Educación con } \\
\text { opción en Administración y Planeación } \\
\text { Educativa }\end{array}$ & 6 & 9 & 345 \\
\hline Total & 60 & 196 & 7,976 \\
\hline
\end{tabular}

Luego de obtener la muestra de cada población, se desarrolló cada uno de los estratos, utilizando la fórmula de Daniels Wayne (1993), con parámetros de confiabilidad de 95\%, 
de error muestral 5\%, y de probabilidad 50\%. La muestra probabilística estratificada estuvo compuesta por 50 directivos/gestores (gráfica 1), 130 profesores (gráfica 2) y 367 estudiantes (gráfica 3).

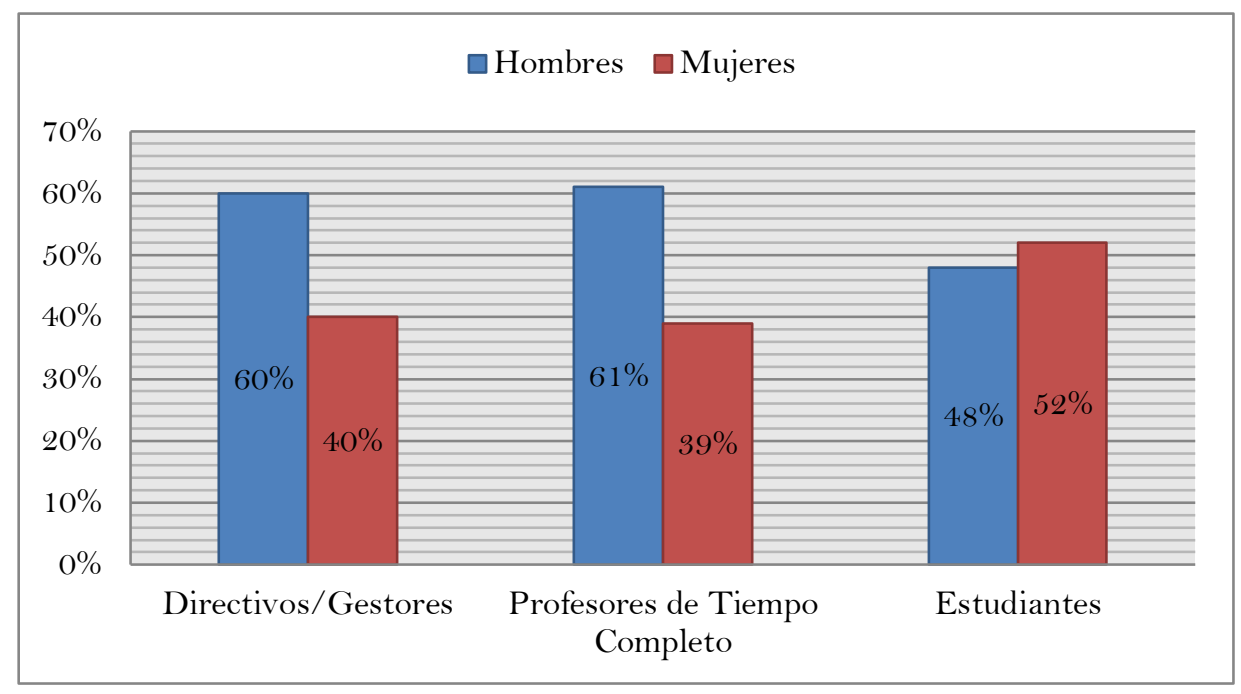

Gráfica 1. Sexo de los sujetos encuestados Fuente: Elaboración propia.

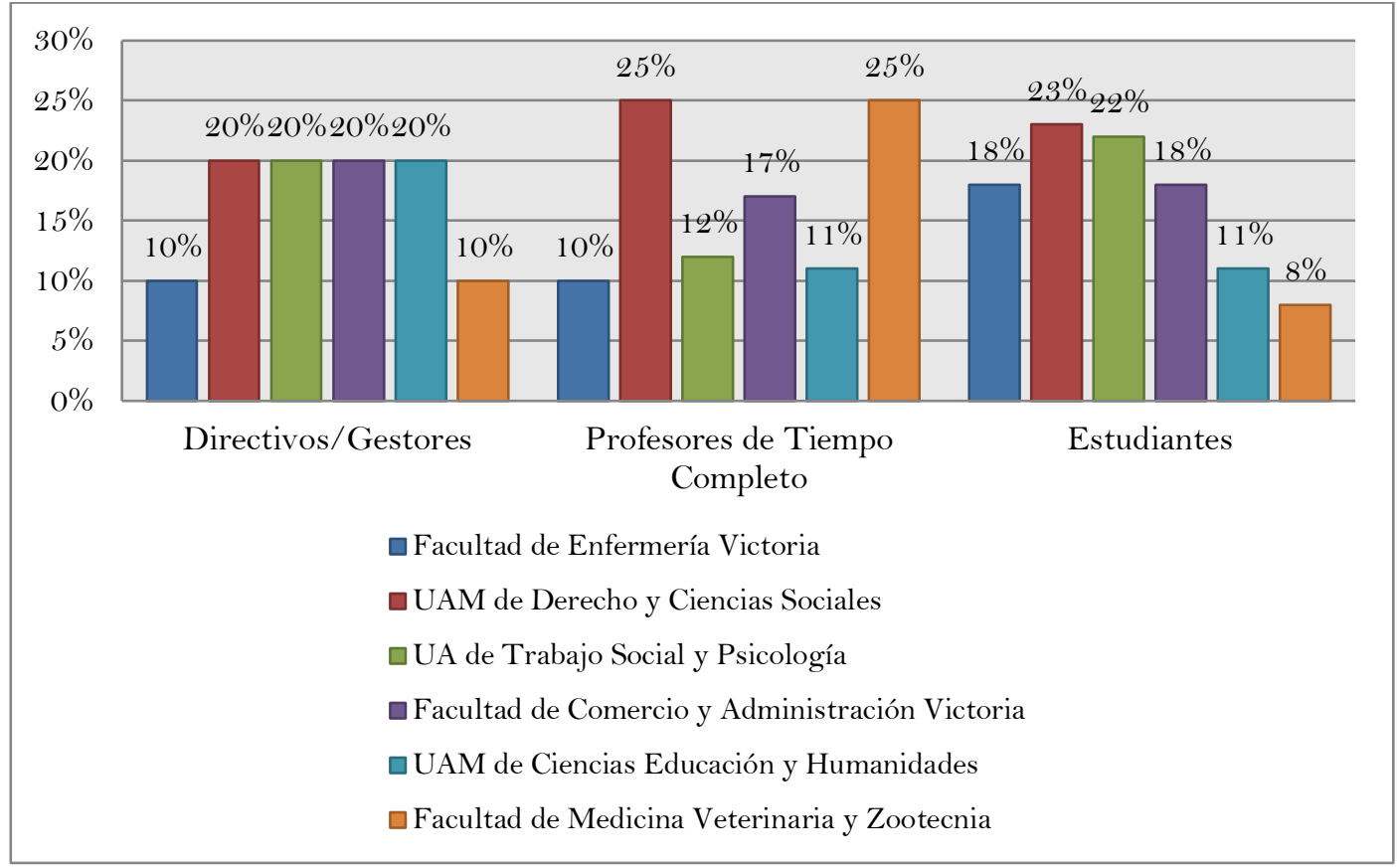

Gráfica 2. Directivos/gestores, PTC y estudiantes según Facultad/Unidad Académica Fuente: Elaboración propia. 


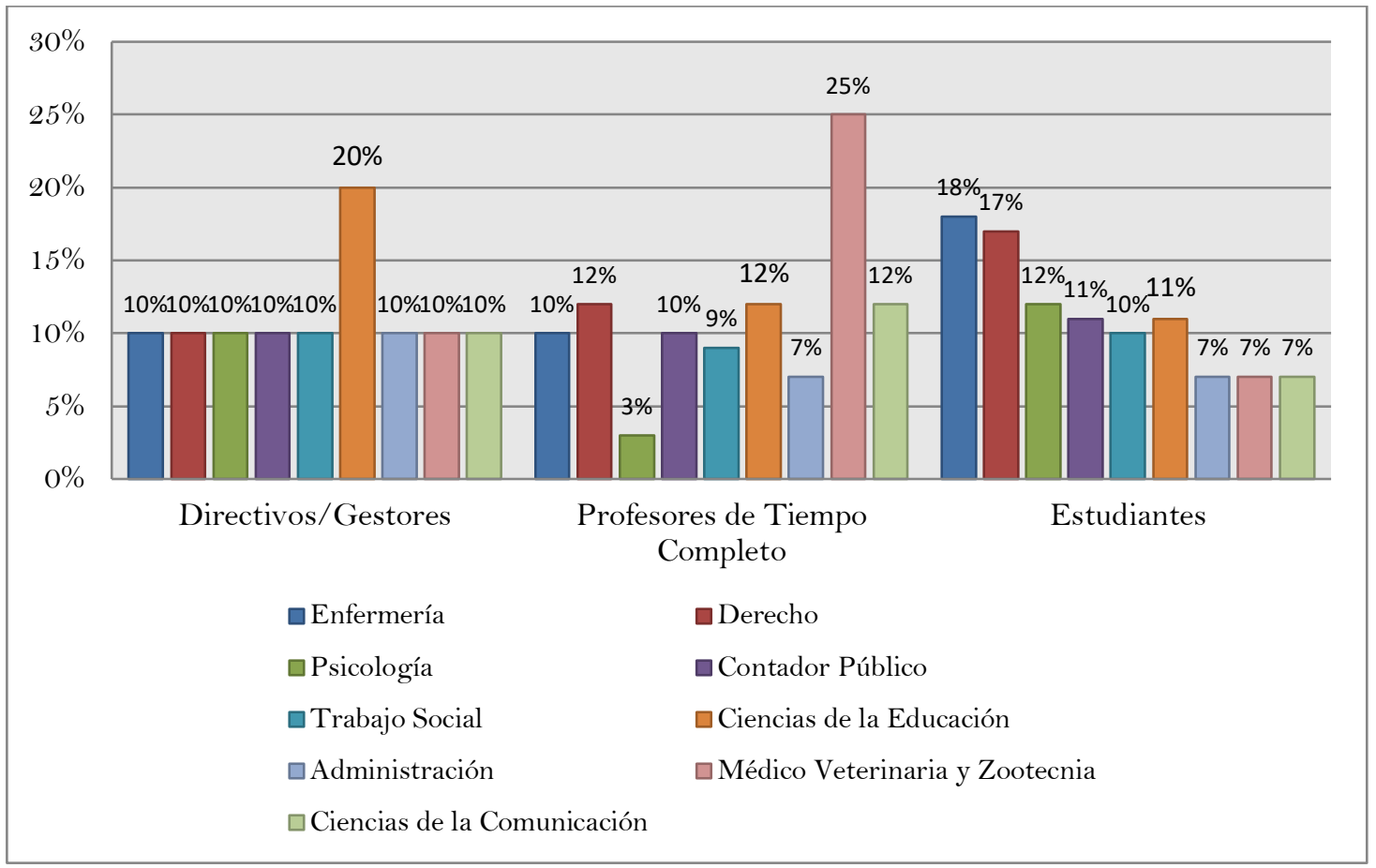

Gráfica 3. Directivos/gestores, PTC y estudiantes según programa educativo Fuente: Elaboración propia.

\section{Resultados}

Los resultados del análisis de la percepción de los directivos gestores, PTC y estudiantes de la UAT sobre el impacto de la acreditación en la mejora de la calidad de los PE se muesntran em las tablas 2,3 y 4 respectivamente.

Los resultados de la investigación dieron evidencias concretas sobre que tanto han impactado en la mejora del trabajo de los directivos y gestores, el desempeño de los profesores, la formación del estudiantado, los esfuerzos realizados para el reconocimiento social de la calidad de los programas de licenciatura, proporcionando un balance preliminar de logros y retos para seguir avanzando en las respuestas sociales que desde la Universidad Autónoma de Tamaulipas espera la sociedad tamaulipeca y en general la mexicana. 
Tabla 2. Percepción de los Directivos/Gestores en relación al impacto de la acreditación en la calidad de los PE

\begin{tabular}{|c|c|c|c|}
\hline DIRECTORES/GESTORES & MuCHO & Poco & NADA \\
\hline \multicolumn{4}{|l|}{ Calidad } \\
\hline Ha cambiado el concepto de calidad que asume la institución educativa. & $80 \%$ & $18 \%$ & $1 \%$ \\
\hline $\begin{array}{l}\text { Ha cambiado la manera en que los miembros participan en la construcción de } \\
\text { la calidad que la institución requiere. }\end{array}$ & $76 \%$ & $24 \%$ & $\mathrm{O} \%$ \\
\hline $\begin{array}{l}\text { Ha cambiado el concepto de calidad adecuándolo a las necesidades del } \\
\text { contexto (en términos económicos, sociales y políticos) en los ámbitos } \\
\text { local, regional, nacional e internacional. }\end{array}$ & $62 \%$ & $38 \%$ & $\mathrm{O} \%$ \\
\hline $\begin{array}{l}\text { Ha cambiado los fines propuestos en la naturaleza, misión y visión de la } \\
\text { institución. }\end{array}$ & $78 \%$ & $22 \%$ & $0 \%$ \\
\hline $\begin{array}{l}\text { Ha cambiado la readaptación continua de la institución a su multientorno } \\
\text { social, cultural y político. }\end{array}$ & $62 \%$ & $34 \%$ & $6 \%$ \\
\hline \multicolumn{4}{|l|}{ Calidad Educativa } \\
\hline Ha cambiado el posicionamiento del PE en su medio. & $86 \%$ & $14 \%$ & $\mathrm{O} \%$ \\
\hline Ha cambiado los mecanismos de vinculación con empleadores y egresados. & $66 \%$ & $33 \%$ & $2 \%$ \\
\hline $\begin{array}{l}\text { Ha cambiado la normativa del PE haciéndola más coherente con las } \\
\text { normativas de la DES y de la Universidad. }\end{array}$ & $76 \%$ & $20 \%$ & $4 \%$ \\
\hline $\begin{array}{l}\text { Ha cambiado los medios para alcanzar las metas establecidas en el plan de } \\
\text { desarrollo del PE, de la DES y de la Universidad. }\end{array}$ & $86 \%$ & $14 \%$ & $0 \%$ \\
\hline $\begin{array}{l}\text { Ha mejorado la planta académica del } \mathrm{PE} \text {, en términos de competitividad y } \\
\text { capacidad académica. }\end{array}$ & $74 \%$ & $24 \%$ & $2 \%$ \\
\hline Ha mejorado las tasas de retención de los estudiantes. & $56 \%$ & $36 \%$ & $8 \%$ \\
\hline \multicolumn{4}{|l|}{ Gestión Institucional } \\
\hline Ha fomentado el trabajo colaborativo entre los miembros del PE. & $70 \%$ & $30 \%$ & $\mathrm{O} \%$ \\
\hline $\begin{array}{l}\text { Ha cambiado la forma de trabajar, integrando a los miembros de la institución } \\
\text { para realizar actividades que permitan cumplir con los objetivos y metas } \\
\text { establecidos en el plan de desarrollo del PE, la DES y la Universidad. }\end{array}$ & $70 \%$ & $30 \%$ & $\mathrm{O} \%$ \\
\hline Ha cambiado la misión y visión del PE, de la DES y de la Universidad. & $54 \%$ & $42 \%$ & $4 \%$ \\
\hline $\begin{array}{l}\text { Ha cambiado en los miembros del PE y de la DES el valor que se le tiene al } \\
\text { aprendizaje. }\end{array}$ & $58 \%$ & $40 \%$ & $2 \%$ \\
\hline $\begin{array}{l}\text { Ha fomentado la participación de los miembros de la DES y del PE en la toma } \\
\text { de decisiones. }\end{array}$ & $66 \%$ & $34 \%$ & $\mathrm{O} \%$ \\
\hline Ha cambiado la comunicación y flujo de información. & $70 \%$ & $22 \%$ & $8 \%$ \\
\hline $\begin{array}{l}\text { Ha cambiado la forma de trabajo en la institución generando la autogestión } \\
\text { entre sus miembros. }\end{array}$ & $58 \%$ & $40 \%$ & $2 \%$ \\
\hline \multicolumn{4}{|l|}{ Gestión de la Calidad } \\
\hline Ha cambiado la capacidad de los miembros del PE como agentes de cambio. & $54 \%$ & $46 \%$ & $\mathrm{O} \%$ \\
\hline $\begin{array}{l}\text { Ha cambiado al enfoque prospectivo, fundamentando su análisis y operación } \\
\text { en el presente y en el futuro. }\end{array}$ & $60 \%$ & $36 \%$ & $4 \%$ \\
\hline Ha mejorado la gestión educativa. & $72 \%$ & $28 \%$ & $\mathrm{O} \%$ \\
\hline Ha cambiado los procesos estructurándolos de abajo hacia arriba. & $54 \%$ & $40 \%$ & $6 \%$ \\
\hline $\begin{array}{l}\text { Ha cambiado el estilo de evaluar el trabajo que desempeñan los miembros del } \\
\text { PE, asumiendo un papel más colaborativo. }\end{array}$ & $70 \%$ & $26 \%$ & $4 \%$ \\
\hline $\begin{array}{l}\text { Ha fomentado la reflexión sobre lo que se está haciendo sobre un modelo de } \\
\text { calidad. }\end{array}$ & $74 \%$ & $24 \%$ & $2 \%$ \\
\hline Ha cambiado el uso de los datos utilizándolos de manera constructiva. & $58 \%$ & $40 \%$ & $2 \%$ \\
\hline Ha mejorado la docencia. & $60 \%$ & $36 \%$ & $4 \%$ \\
\hline Ha incrementado la participación de los profesores en la investigación. & $48 \%$ & $52 \%$ & $\mathrm{O} \%$ \\
\hline Ha incrementado el número de cuerpos académicos y grupos disciplinarios. & $44 \%$ & $48 \%$ & $8 \%$ \\
\hline Ha incrementado el número de publicaciones científicas. & $38 \%$ & $62 \%$ & $0 \%$ \\
\hline Ha cambiado los proyectos con impacto social. & $52 \%$ & $42 \%$ & $6 \%$ \\
\hline Ha fomentado la generación de incubadoras de empresas y patentes. & $22 \%$ & $58 \%$ & $20 \%$ \\
\hline $\begin{array}{l}\text { Ha mejorado las instalaciones de la DES para poder desarrollar las } \\
\text { actividades propias del PE. }\end{array}$ & $76 \%$ & $22 \%$ & $2 \%$ \\
\hline $\begin{array}{l}\text { Ha incrementado el compromiso con la labor que desempeña de } \\
\text { director/gestor de la DES y del PE. }\end{array}$ & $88 \%$ & $10 \%$ & $2 \%$ \\
\hline $\begin{array}{l}\text { Ha cambiado la manera en el que los miembros del PE se involucran con los } \\
\text { problemas que afecta a la sociedad. }\end{array}$ & $64 \%$ & $30 \%$ & $6 \%$ \\
\hline $\begin{array}{l}\text { Ha incrementado los convenios de colaboración con instituciones públicas y } \\
\text { privadas. }\end{array}$ & $66 \%$ & $32 \%$ & $2 \%$ \\
\hline Ha mejorado el servicio que ofrece el $\mathrm{PE}$ al que pertenece. & $86 \%$ & $14 \%$ & $0 \%$ \\
\hline
\end{tabular}

Fuente: Elaboración propia con base en resultados de Cuestionario para Directivos/Gestores. 
Tabla 3. Percepción de los PTC en relación al impacto de la acreditación en la calidad de los PE

\begin{tabular}{|c|c|c|c|}
\hline PROFESORES DE TIEMPO COMPLETO & MUCHO & Poco & NADA \\
\hline \multicolumn{4}{|l|}{ Calidad } \\
\hline $\begin{array}{l}\text { Ha cambiado el concepto de calidad propio del PE y de la Unidad } \\
\text { Académica/Facultad. }\end{array}$ & $52 \%$ & $41 \%$ & $7 \%$ \\
\hline $\begin{array}{l}\text { Ha fomentado la participación e involucramiento de los profesores en la } \\
\text { construcción del concepto de calidad propio del PE y de la Unidad } \\
\text { Académica/Facultad. }\end{array}$ & $46 \%$ & $49 \%$ & $5 \%$ \\
\hline $\begin{array}{l}\text { Ha cambiado el concepto de calidad adecuándolo a las necesidades del } \\
\text { contexto (en términos económicos, sociales y políticos) en los ámbitos } \\
\text { local, regional, nacional e internacional. }\end{array}$ & $43 \%$ & $49 \%$ & $8 \%$ \\
\hline \multicolumn{4}{|l|}{ Calidad Educativa } \\
\hline Ha cambiado el posicionamiento del PE en su medio. & $52 \%$ & $38 \%$ & $10 \%$ \\
\hline Ha cambiado la vinculación de la investigación con la docencia. & $35 \%$ & $53 \%$ & $12 \%$ \\
\hline Ha mejorado las calificaciones de los estudiantes. & $21 \%$ & $65 \%$ & $14 \%$ \\
\hline Ha mejorado los niveles de aprendizaje de los estudiantes. & $27 \%$ & $61 \%$ & $12 \%$ \\
\hline \multicolumn{4}{|l|}{ Gestión Institucional } \\
\hline Ha fomentado la participación de los profesores en la toma decisiones del PE. & $43 \%$ & $46 \%$ & $11 \%$ \\
\hline Ha fomentado el trabajo colaborativo entre los profesores. & $42 \%$ & $45 \%$ & $13 \%$ \\
\hline $\begin{array}{l}\text { Ha mejorado tus capacidades para solucionar problemas complejos al interior } \\
\text { de la Universidad, en su profesión y los que se generan en tu vida diaria } \\
\text { en sociedad. }\end{array}$ & $43 \%$ & $43 \%$ & $14 \%$ \\
\hline $\begin{array}{l}\text { Ha mejorado la comunicación y flujo de información manteniendo enterados a } \\
\text { los profesores sobre cuestiones del PE. }\end{array}$ & $47 \%$ & $43 \%$ & $10 \%$ \\
\hline Ha fomentado la autogestión en los profesores. & $32 \%$ & $59 \%$ & $9 \%$ \\
\hline $\begin{array}{l}\text { Ha mejorado los mecanismos de evaluación docente (estudiantes a profesores, } \\
\text { profesores a profesores e institución a profesores). }\end{array}$ & $45 \%$ & $46 \%$ & $9 \%$ \\
\hline \multicolumn{4}{|l|}{ Gestión de la Calidad } \\
\hline $\begin{array}{l}\text { Ha fomentado en el profesor la libertad de definir sus objetivos de calidad y } \\
\text { diseñar el proceso de aprendizaje. }\end{array}$ & $42 \%$ & $46 \%$ & $12 \%$ \\
\hline $\begin{array}{l}\text { Ha cambiado la capacidad del profesor considerándolo un agente de cambio } \\
\text { tanto individual como colectivamente. }\end{array}$ & $33 \%$ & $55 \%$ & $12 \%$ \\
\hline $\begin{array}{l}\text { Ha cambiado las prácticas docentes acorde a las características particulares } \\
\text { del PE. }\end{array}$ & $38 \%$ & $53 \%$ & $8 \%$ \\
\hline $\begin{array}{l}\text { Ha fomentado la aplicación de estrategias de evaluación que permiten } \\
\text { verificar el cumplimiento de los objetivos de aprendizaje en forma } \\
\text { continua. }\end{array}$ & $42 \%$ & $50 \%$ & $8 \%$ \\
\hline $\begin{array}{l}\text { Ha cambiado los métodos de evaluación que se aplican a los estudiantes, a } \\
\text { manera de ser congruentes con los objetivos del plan de estudios del PE. }\end{array}$ & $47 \%$ & $42 \%$ & $11 \%$ \\
\hline Ha mejorado las condiciones de trabajo del profesorado. & $38 \%$ & $45 \%$ & $18 \%$ \\
\hline Ha incrementado los apoyos administrativos a los profesores. & $41 \%$ & $41 \%$ & $18 \%$ \\
\hline Ha incrementado la participación de los profesores al programa de tutorías. & $39 \%$ & $48 \%$ & $12 \%$ \\
\hline Ha mejorado la forma de trabajar y rendimiento de las academias. & $34 \%$ & $48 \%$ & $18 \%$ \\
\hline Ha cambiado los cursos de capacitación para los profesores. & $43 \%$ & $44 \%$ & $13 \%$ \\
\hline Ha fomentado la participación de los profesores en la investigación. & $28 \%$ & $56 \%$ & $15 \%$ \\
\hline Ha mejorado la forma y rendimiento de trabajo de los cuerpos académicos. & $32 \%$ & $52 \%$ & $15 \%$ \\
\hline $\begin{array}{l}\text { Ha fomentado las redes de colaboración con otros cuerpos académicos a nivel } \\
\text { institucional, estatal, nacional e internacional. }\end{array}$ & $34 \%$ & $48 \%$ & $18 \%$ \\
\hline $\begin{array}{l}\text { Ha fomentado la participación de los profesores en las publicaciones } \\
\text { científicas. }\end{array}$ & $31 \%$ & $55 \%$ & $14 \%$ \\
\hline Ha incrementado la participación de profesores en el asesoramiento de tesis. & $37 \%$ & $48 \%$ & $15 \%$ \\
\hline $\begin{array}{l}\text { Ha cambiado el compromiso de los profesores en la formación de } \\
\text { profesionales. }\end{array}$ & $54 \%$ & $35 \%$ & $11 \%$ \\
\hline $\begin{array}{l}\text { Ha incrementado la participación de los profesores en programas sociales de } \\
\text { ayuda a la comunidad. }\end{array}$ & $26 \%$ & $55 \%$ & $19 \%$ \\
\hline $\begin{array}{l}\text { Ha fomentado la participación de los profesores en la divulgación del } \\
\text { conocimiento (conferencias, pláticas de interés social, paneles, etc.) } \\
\text { dirigidos a la sociedad. }\end{array}$ & $33 \%$ & $57 \%$ & $10 \%$ \\
\hline Ha mejorado el servicio educativo que ofrece el PE. & $48 \%$ & $42 \%$ & $10 \%$ \\
\hline
\end{tabular}

Fuente: Elaboración propia con base en resultados de Cuestionario para Profesores de Tiempo Completo. 
Tabla 4. Percepción de los Estudiantes en relación al impacto de la acreditación en la calidad de los PE

\begin{tabular}{lcccc}
\hline \multicolumn{1}{c}{ EsTUDIANTES } & MUCHO & POCO & NADA & NC \\
\hline Calidad & & & & \\
\hline $\begin{array}{l}\text { Ha cambiado el concepto de calidad. } \\
\text { Ha fomentado la participación de los estudiantes en la construcción }\end{array}$ & $49 \%$ & $48 \%$ & $2 \%$ & $0 \%$ \\
$\begin{array}{l}\text { del concepto de calidad propio del PE y de la Unidad } \\
\text { Académica/Facultad. }\end{array}$ & $50 \%$ & $45 \%$ & $5 \%$ & $0 \%$ \\
$\begin{array}{l}\text { Ha mejorado la respuesta y acoplamiento continuo del PE y la } \\
\text { Unidad Académica/Facultad a las necesidades que existen en el }\end{array}$ & $52 \%$ & $43 \%$ & $5 \%$ & $0 \%$ \\
ámbito social, cultural y político. & & & & \\
\hline
\end{tabular}

\section{Calidad Educativa}

Ha cambiado el posicionamiento, reconocimiento y prestigio del PE en su medio como un programa líder en el sector educativo y en la sociedad.

Ha cambiado el acercamiento del PE a la comunidad y al sector productivo.

Ha cambiado la coherencia entre lo que se ofrece como parte del PE y lo que realmente se da.

Ha fomentado la participación de los estudiantes en la investigación.

Ha mejorado el rendimiento académico de los estudiantes.

\section{Gestión Institucional}

Ha fomentado la participación de los estudiantes en la toma decisiones del PE.

Ha fomentado el trabajo colaborativo entre los estudiantes.

Ha mejorado tus capacidades para solucionar problemas complejos al interior de la Universidad, de tu carrera profesional y los que se generan en tu vida diaria en sociedad.

Ha mejorado la comunicación y flujo de información, manteniendo enterados a los estudiantes sobre cuestiones del PE vinculados a ustedes.

Ha fomentado en los estudiantes la gestión de su propio aprendizaje y del conocimiento. Gestión de la Calidad
Ha cambiado la capacidad de los estudiantes como agentes de
cambio tanto individual como colectivamente.

Ha cambiado las estrategias de evaluación de los profesores aplicados a los estudiantes permitiendo verificar el cumplimiento de los objetivos de aprendizaje en forma continua.

Ha cambiado las condiciones del aula a manera de propiciar el proceso de aprendizaje.

Ha mejorado el aula, incorporando las TICs como parte del proceso del proceso educativo.

Ha fortalecido el programa de asesorías académicas.

Ha fortalecido el programa de tutorías.

Ha incrementado el número del acervo bibliográfico y revistas especializadas.

Ha mejorado las condiciones del auditorio para desarrollar eventos y actividades académicas.

Ha incrementado el número de computadoras con acceso a internet para uso de los estudiantes en el centro de cómputo.

Ha facilitado el acceso libre a internet (wifi) permitiendo acceder desde cualquier espacio de la Unidad Académica/Facultad.

Ha incrementado la promoción de cursos extracurriculares.

Ha propiciado la participación de los estudiantes a los distintos grupos deportivos, culturales y artísticos de la Unidad Académica/Facultad.

Ha incrementado la participación de los estudiantes en realización de tesis.

Ha incrementado la participación de los estudiantes en investigaciones que realizan los profesores del PE.

Ha incrementado la participación de los estudiantes en estancias de investigación en otras universidades del país o en el extranjero.

\begin{tabular}{|c|c|c|c|}
\hline $48 \%$ & $46 \%$ & $6 \%$ & $0 \%$ \\
\hline $42 \%$ & $50 \%$ & $8 \%$ & $0 \%$ \\
\hline $34 \%$ & $55 \%$ & $10 \%$ & $0 \%$ \\
\hline $44 \%$ & $46 \%$ & $9 \%$ & $1 \%$ \\
\hline $51 \%$ & $42 \%$ & $6 \%$ & $1 \%$ \\
\hline $33 \%$ & $53 \%$ & $14 \%$ & $1 \%$ \\
\hline $51 \%$ & $41 \%$ & $7 \%$ & $0 \%$ \\
\hline $47 \%$ & $49 \%$ & $9 \%$ & $0 \%$ \\
\hline $41 \%$ & $49 \%$ & $9 \%$ & $1 \%$ \\
\hline $47 \%$ & $45 \%$ & $8 \%$ & $0 \%$ \\
\hline $39 \%$ & $53 \%$ & $8 \%$ & $0 \%$ \\
\hline $49 \%$ & $44 \%$ & $7 \%$ & $\mathrm{O} \%$ \\
\hline $44 \%$ & $43 \%$ & $12 \%$ & $1 \%$ \\
\hline $46 \%$ & $41 \%$ & $11 \%$ & $1 \%$ \\
\hline $41 \%$ & $44 \%$ & $14 \%$ & $1 \%$ \\
\hline $33 \%$ & $51 \%$ & $15 \%$ & $1 \%$ \\
\hline $37 \%$ & $47 \%$ & $14 \%$ & $1 \%$ \\
\hline $45 \%$ & $44 \%$ & $11 \%$ & \\
\hline $43 \%$ & $41 \%$ & $15 \%$ & $1 \%$ \\
\hline $39 \%$ & $36 \%$ & $24 \%$ & $1 \%$ \\
\hline $66 \%$ & $19 \%$ & $14 \%$ & $1 \%$ \\
\hline $48 \%$ & $40 \%$ & $11 \%$ & $1 \%$ \\
\hline $35 \%$ & $52 \%$ & $12 \%$ & $1 \%$ \\
\hline $38 \%$ & $51 \%$ & $11 \%$ & $1 \%$ \\
\hline $40 \%$ & $48 \%$ & $11 \%$ & $1 \%$ \\
\hline
\end{tabular}

Fuente: Elaboración propia con base en resultados de Cuestionario para Estudiantes. 
Tabla 5. Percepción de los Estudiantes en relación al impacto de la acreditación en la calidad de los PE (continuación)

\begin{tabular}{|c|c|c|c|c|}
\hline ESTUDIANTES & MUCHO & Poco & NADA & NC \\
\hline \multicolumn{5}{|l|}{ Gestión de la Calidad } \\
\hline $\begin{array}{l}\text { Ha cambiado tu compromiso como ciudadano, alumno y futuro } \\
\text { profesional. }\end{array}$ & $55 \%$ & $36 \%$ & $8 \%$ & $1 \%$ \\
\hline $\begin{array}{l}\text { Ha incrementado la participación de los estudiantes en programas } \\
\text { sociales de ayuda a la comunidad. }\end{array}$ & $56 \%$ & $38 \%$ & $5 \%$ & $0 \%$ \\
\hline $\begin{array}{l}\text { Ha fomentado la participación de los estudiantes en la divulgación } \\
\text { del conocimiento (conferencias, pláticas de interés social, } \\
\text { paneles, etc.). }\end{array}$ & $44 \%$ & $47 \%$ & $9 \%$ & $0 \%$ \\
\hline Ha mejorado el servicio educativo que ofrece el PE. & $51 \%$ & $39 \%$ & $9 \%$ & $0 \%$ \\
\hline
\end{tabular}

Fuente: Elaboración propia con base en resultados de Cuestionario para Estudiantes.

\subsection{Calidad}

Los directivos/gestores consideran que la acreditación ha cambiado mucho el concepto de calidad que asume la institución educativa, los fines propuestos en la naturaleza, misión y visión de la institución, la manera en que los miembros participan en la construcción de la calidad que la institución requiere, sin embargo, no ha impactado de forma significativa en una calidad que responda a las necesidades del contexto (en términos económicos, sociales y políticos) en los ámbitos local, regional, nacional e internacional, así como en la readaptación continua de la institución a su multientorno social, cultural y político. Mientras que los docentes al igual que los estudiantes perciben que la acreditación ha impactado parcialmente en el concepto de calidad propio del PE y de la Unidad Académica/Facultad, la participación e involucramiento de los profesores en la construcción de calidad educativa y su adecuación a las necesidades del contexto.

\subsection{Calidad Educativa}

Los directivos/gestores consideran que la acreditación ha impactado significativamente en el posicionamiento, reconocimiento y prestigio de la carrera en su contexto, en la coherencia entre normativa del PE, de la DES y de la Universidad, en los medios para alcanzar las metas establecidas en el plan de desarrollo, y los mecanismos de vinculación con empleadores y egresados, en la capacidad y competitividad académica de sus docentes, así como las tasas de retención de los estudiantes. Los docentes reconocen que la acreditación ha logrado cambios institucionales en el reconocimiento del programa educativo en su medio; sin embargo, poco ha cambiado la vinculación de la investigación con la docencia y poco ha mejorado las calificaciones de los estudiantes y los niveles de aprendizaje. Por su parte, los estudiantes consideran que la acreditación ha impactado parcialmente en el posicionamiento, reconocimiento y prestigio del $\mathrm{PE}$ en su medio como un programa líder en el sector educativo y en la sociedad, en el acercamiento del PE a la comunidad y al sector productivo, en la coherencia entre lo que se ofrece como parte del PE y lo que realmente se da en su rendimiento académico y en la participación de los estudiantes en la investigación.

\subsection{Gestión institucional}

Los directivos/gestores, consideran que la acreditación ha impactado de forma significativa en participación, toma de decisiones y trabajo colaborativo entre los miembros de la institucional, la comunicación y flujo de información, ha generado cambios de relevancia en la misión y visión de la carrera e institucional, en las 
capacidades para solucionar problemas complejos que se generan en la institución, y en el valor que se le tiene al aprendizaje y al conocimiento, como parte importante de la vida institucional. En los profesores, hay una percepción variada del impacto de la acreditación en la mejora de las capacidades para solucionar problemas complejos al interior de la universidad, en su profesión y los que se generan en su vida diaria en sociedad. Reconocen cambios institucionales en la comunicación y flujo de información, sin embargo, la acreditación poco ha fomentado la participación de los profesores en la toma decisiones del programa educativo, el trabajo colaborativo entre los docentes, impulsando el trabajo en equipo, la autogestión en los profesores y poco ha mejorado los mecanismos de evaluación docente. Por su parte, los estudiantes consideran que la acreditación ha impactado de forma significativa en el ambiente y proceso educativo centrándose en el alumno y en el aprendizaje, en el trabajo colaborativo, impulsando el trabajo en equipo y la autogestión en ellos (ser capaces individualmente de construir el conocimiento y ser autodidactas de su propio aprendizaje), en las competencias para solucionar problemas individuales, profesionales y de su contexto; sin embargo, poco se ha fomentado la participación de los estudiantes en la toma decisiones del programa educativo, y poco se ha propiciado la comunicación y flujo de información.

\subsection{Gestión de la calidad}

Los directivos/gestores, consideran que la acreditación ha impactado de forma significativa en el enfoque prospectivo de la gestión, el estilo de evaluar el trabajo que desempeñan los académicos, asumiendo un papel más colaborativo, los procesos estructurándolos de abajo hacia arriba, el uso de la información utilizándolos de manera constructiva, la manera en el que los académicos y administrativos se involucran con problemas que afecta a la sociedad y el desarrollo de proyectos de investigación con impacto social, la capacidad de los miembros del programa educativo considerándolos como agentes de cambio tanto individual como colectivamente. La acreditación ha generado cambios institucionales en la reflexión sobre lo que se está haciendo en un modelo de calidad, la gestión educativa y la docencia, el servicio educativo, la vinculación estratégica con el entorno y el compromiso como director/gestor con la institución. Se reconoce que poco ha incrementado la participación de los profesores en la investigación, el número de cuerpos académicos y grupos disciplinarios, publicaciones científicas, y poco se ha fomentado la generación de incubadoras de empresas y patentes.

Los docentes consideran que la acreditación ha mejorado mucho el servicio educativo, su compromiso como profesor en la formación de profesionales; sin embargo, poco ha incrementado la aplicación de estrategias de evaluación que permiten verificar el cumplimiento de los objetivos de aprendizaje en forma continua, la participación de profesores en la investigación, programas de tutorías y programas sociales de ayuda a la comunidad, poco ha cambiado las prácticas docentes, los cursos de capacitación para los profesores, poco ha mejorado las condiciones de su trabajo, la forma de trabajar y rendimiento de las academias.

Finalmente, los estudiantes perciben que la acreditación ha impactado de forma significativa en el servicio educativo, su compromiso como ciudadano, alumno y futuro profesional, en las condiciones tanto de infraestructura física y tecnológica del aula propiciando el proceso de aprendizaje, en la aplicación de estrategias de evaluación por parte de los profesores dando un seguimiento puntual al cumplimiento de los objetivos 
de aprendizaje en forma continua, en la promoción de cursos extracurriculares, participación en programas sociales de ayuda a la comunidad, y en distintos grupos deportivos, culturales y artísticos. Sin embargo, poco se ha fortalecido el programa de tutorías y de asesorías académicas, poco se ha incrementado el número del acervo bibliográfico y revistas especializadas, impresas y electrónicas, poco ha fomentado la participación de los estudiantes en la divulgación del conocimiento, en la realización de tesis y en estancias de investigación, y poco ha cambiado la capacidad de los estudiantes como agentes de cambio en su contexto.

\section{Discusión y conclusiones}

Aunque la acreditación ha tenido avances significativos a su llegada a la educación superior (Buendía, 2014) los resultados obtenidos en el proyecto del CINDA en México, muestran que los procesos de acreditación no han generado efectos decisivos en la mejora de la calidad de los PE debido a la influencia de otros factores. Si bien se ha avanzado en una cultura de la evaluación (De la Garza, 2013), esta aceptación se podría explicar a partir de las implicaciones financieras, actualmente las IES evalúan sus PE para mejorar, pero también para obtener recursos económicos ya que las políticas de financiamiento apuntan hacía la medición de indicadores de calidad, generando algunos cambios institucionales a favor del servicio educativo que se ofrece (Castillo, 2004).

El estudio realizó una aportación significativa al campo del conocimiento en materia de calidad educativa, como un caso de estudio de una universidad del noreste de México que ha mostrado signos de esfuerzos en conjunto y características particulares del enfoque del mejoramiento de la calidad, lo que le ha permitido consolidar sus PE, recibiendo el reconocimiento público de calidad a través de los diversos organismos acreditadores. Cabe aclarar que os resultados de esta investigación no pueden ser generalizados debido a que se trata de un estudio de caso que presenta diferentes características y particularidades propias de la UAT, que la hacen diferente a la naturaleza de otras IES.

Desde las percepciones de los directivos/gestores y profesorado de tiempo completo, que se indagaron con base en los trabajos coordinados por Antonio Cardona (2011) y estudiantes de los programas educativos acreditados de la UAT, la acreditación contribuye parcialmente a la mejora de la calidad de las carreras acreditadas que ofrece la institución, crea cambios institucionales que pueden contribuir a asegurar la sustentabilidad de la calidad educativa, mantener el compromiso social de la universidad, produce conocimiento útil, pertinente e innovador y contribuye al tránsito hacia una de las sociedades del conocimiento, aunque estos efectos no son suficientes para mejorar permanentemente la calidad (CIAC, 2012), por lo que se necesitará replantear el actual esquema de acreditación institucional.

Sin embargo, existen algunas diferencias entre las opiniones de los actores las cuales dependen de las posiciones y el rol que desempeñan, por lo que se necesita continuar profundizando este tipo de investigaciones para proveer a quienes toman decisiones institucionales y de políticas públicas, de elementos concretos y evidencias sobre las cuales avanzar en el mejoramiento de la calidad en la educación superior.

Será necesario que la universidad supere algunas tareas pendientes como reconocer y cumplir plenamente la misión encomendada por la sociedad, garantizar la calidad de su 
oferta educativa, articular y cerrar brechas de las funciones sustantivas y procesos de gestión con los resultados educativos no solo cuantitativos sino también cualitativos, mejorar las formas de gobierno universitario involucrando al conjunto de los actores, responder a las necesidades de profesores y estudiantes, priorizando el aprendizaje incluyendo algunos aspectos a trabajar sobre gestión de recursos, trabajo colaborativo y participativo, formación profesional y ciudadana, innovación en los procesos académicos y administrativos, investigación útil y pertinente al contexto, infraestructura, tecnología aplicada al trabajo y procesos de aprendizaje, internacionalización de los programas educativos, asumir el compromiso total de la responsabilidad social, la transparencia y rendición de cuentas, entre otros los cuales deberán ser incorporados en las políticas y actuaciones institucionales.

\section{Referencias}

ANUIES. (2000). La educación superior en el siglo XXI. Líneas estratégicas de desarrollo. Propuesta de la ANUIES. Ciudad de México: ANUIES.

Brennan, J. (1998). Panorama general del aseguramiento de la calidad. En S. Malo y A. Velásquez (Coord.), La calidad en la educación superior en México (pp. 13-30). Ciudad de México: Porrúa.

Buendía, A. (2014). Evaluación y acreditación de programas académicos en México: Revisar los discursos, valorar los efectos. Ciudad de México: ANUIES.

Cardona, A. (2011). Calidad en la educación superior. ¿Qué modelo y en qué condiciones? La opinión del profesorado en Argentina, España y México. Guadalajara: Editorial Universitaria.

CINDA. (2012). Aseguramiento de la calidad en Iberoamérica. Educación Superior. Informe. Santiago de Chile: RL Editores.

Congreso Internacional de Acreditación y Certificación de Programas e Instituciones de Educación Superior (CIAC). (2012). Memorias en Extenso. Puerto Vallarta: Consejo para la Acreditación de la Educación Superior y la Red de Académicos de Iberoamérica.

Cruz, Y., Escrigas, C., López, F., Sanyal, B. y Tres, J. (2007). Prólogo: La acreditación para la garantía de la calidad y el compromiso social de las universidades: ¿Qué está en juego? En Sanyal, B. y Tres, J. (Eds.), En la Educación Superior en el mundo 2007. La acreditación para la garantía de la calidad: ¿Qué está en juego? (pp. 28-48). Barcelona: University Network for Innovation (GUNI).

De la Garza, J. (2013). La evaluación de programas educativos del nivel superior en México. Avances y perspectivas. Perfiles Educativos, 35, 35-45.

Del Castillo, G. (2004). El impacto de la evaluación externa en dos instituciones de educación superior en México: La Universidad Autónoma Metropolitana Azcapotzalco y la Universidad Iberoamericana. Perfiles Latinoamericanos, 25(diciembre), 115-148.

Días, J. (2012). Políticas y conceptos de calidad: Dilemas y retos. Revista Avaliação da Eduçãcion Superior, 3(17), 601-618. https://doi.org/10.1590/s1414-40772012000300003

Drucker, P. (2004). La sociedad post capitalista. Bogotá: Norma.

Fernández, N. (2004). Hacia la convergencia de los sistemas de educación superior en América Latina. Revista Iberoamericana de Educación, 35(mayo-agosto). Recuperado de http://rieoei.org/rie35a02.htm 
Harvey, L. y Green, D. (1993). Defining quality. Assessment \& Evaluation in Higher Education, 18(1), 9-34. https://doi.org/10.1080/0260293930180102

Hernández, R., Fernández, C. y Baptista, P. (2010). Metodología de la investigación. Ciudad de México: McGraw.

Latapí, P. (2008). Una buena educación. Reflexiones sobre la calidad. Ciudad de México: Universidad de Colima.

Lemaitre, M. (2012). Una mirada actual al desarrollo de procesos de aseguramiento de la calidad. Documentos académicos del Consejo Nacional de Acreditación. Recuperado de http:/ / portal.mec.gov.br/index.php?option=com_docman\&view=download $\&$ alias $=4335$ conae4-08042010\&Itemid $=30192$

Marúm E. y Rosario, V. (Coords.). (2011). Calidad educativa. Concepto, contexto y percepción del profesorado. Ciudad de México: Editorial Universitaria, Universidad de Guadalajara.

Marúm, E. (1995). Avances en el proceso de acreditación de las instituciones de educación superior en México. Revista de la Educación Superior, 96(24). Recuperado de http://publicaciones.anuies.mx/pdfs/revista/Revista96_S1A3ES.pdf

Marúm, E., Mustre, J. y Nieto, L. (2011). Calidad educativa. Un concepto multidimensional. En E. Marúm y V. Rosario (Comp), Calidad Educativa. Concepto, contexto y percepción del profesorado (pp. 19-61). Guadalajara: Editorial Universitaria.

Mintzberg, H. (1997). El trabajo del administrador: Fantasías y realidades. En H. Mintzberg, B. James y H. Voyer (Eds.), El proceso estratégico. Conceptos, contextos y casos (pp. 35-44). Ciudad de México: Prentice Hall.

Namakforoosh, N. (2002). Metodología de la investigación. Ciudad de México: Limusa.

Olaskoaga J. (2012). Universidades en Movimiento. El debate sobre la gestión de la calidad y las actitudes del profesorado ante las transformaciones universitarias. Ciudad de México: ANUIES.

Rosario, V. (2012). Repensar los procesos de acreditación en la universidad pública mexicana en el marco de su responsabilidad social. En V. Rosario, A. Didriksson y E. Marúm (Coord,), La educación de la educación superior en Iberoamérica. La gestión de la calidad de los programas educativos. Tensiones, desencuentros, conflictos y resultados (pp. 195-209). Bloomington, IN: Palibrio.

Senge, P. y Cambron, N. (2002). Escuelas que aprenden. Un manual de la Quinta Disciplina para educadores, padres de familia y todos los que se interesen en la educación. Bogotá: Norma.

UNESCO. (1998). La educación superior en el siglo XXI. Visión y acción. Informe Final de la Conferencia Mundial sobre la Educación Superior París. París: Autor.

Wayne, D. (1993). Estadística con aplicaciones a las ciencias sociales y a la educación. Ciudad de México: Mc Graw Hill.

\section{Breve CV dos autores}

\section{Erik Márquez de León}

Profesor de Tiempo Completo, Coordinador de la Licenciatura en Ciencias de la Educación con acentuación en Evaluación Educativa, y Miembro del Comité para la Acreditación Internacional para la Gestión Institucional de la Universidad Autónoma de Tamaulipas (UAT). Miembro de la Red Internacional de Evaluadores (RIEV). 
Estudiante del Doctorado en Evaluación Educativa por el Instituto para la Medición, Mejoramiento y Aseguramiento de la Calidad de la Educación (IMMACE). Maestro en Gestión y Políticas de la Educación Superior por el Centro Universitario de Ciencias Económico Administrativas de la Universidad de Guadalajara (UdeG), programa adscrito al Padrón Nacional de Posgrados de Calidad del Consejo Nacional de Ciencia y Tecnología (PNPC-CONACyT). Especialista en Planeación y Evaluación Universitaria por el IMMACE. Licenciado en Ciencias de la Educación con opción en Ciencias Sociales por la Unidad Académica Multidisciplinaria de Ciencias, Educación y Humanidades de la UAT. ORCID ID: 0000-0002-1407-0312. Email: ermarquez@docentes.uat.edu.mx

\section{Zulma Raquel Zeballos Pinto}

Facilitadora de la Universidad Da Vinci. Miembro de la Red Internacional de Evaluadores (RIEV). Estudiante del Doctorado en Educación por el Centro Universitario de Ciencias Sociales y Humanidades de la Universidad de Guadalajara (UdeG), programa adscrito al Padrón Nacional de Posgrados de Calidad del Consejo Nacional de Ciencia y Tecnología (PNPC-CONACyT). Maestra en Gestión y Políticas de la Educación Superior por el Centro Universitario de Ciencias Económico Administrativas de la UdeG, programa adscrito al PNPC-CONACyT. Especialista en Planeación y Evaluación Universitaria por el Instituto para la Medición, Mejoramiento y Aseguramiento de la Calidad de la Educación (IMMACE). Licenciada en Ciencias de la Educación con opción en Administración y Planeación Educativa por la Unidad Académica Multidisciplinaria de Ciencias, Educación y Humanidades de la UAT. ORCID ID: 0000-0002-2442-7610. Email: zzeballos@udavinci.edu.mx 